gr-qc/9403008

Imperial/TP/93-94/20

Int. J. Mod. Phys. A10 (1995) 145

\title{
Quantum gravity and minimum length
}

\author{
Luis J. Garay \\ Theoretical Physics Group, Blackett Laboratory, Imperial College, \\ Prince Consort Road, London SW7 2BZ, U.K.
}

(5 August 1994)

\begin{abstract}
The existence of a fundamental scale, a lower bound to any output of a position measurement, seems to be a model-independent feature of quantum gravity. In fact, different approaches to this theory lead to this result. The key ingredients for the appearance of this minimum length are quantum mechanics, special relativity and general relativity. As a consequence, classical notions such as causality or distance between events cannot be expected to be applicable at this scale. They must be replaced by some other, yet unknown, structure.
\end{abstract}

\section{INTRODUCTION}

Quantum gravity, the yet-to-be-built quantum theory of gravity, involves a series of problems (see Ref. [1] for a review) that have remained unsolved for many years. Most of the problems arise from the fact that, unlike any other interaction, gravity deals with the frame in which everything takes place, with spacetime. Quantization of any interaction except gravity leaves this frame unchanged. It is a passive frame. But when gravity is brought onto the scene, the frame itself becomes dynamical. It suffers the quantum fluctuations of the other interactions and, even more, introduces its own fluctuations. It becomes an active agent in the theory. Human mind is used to putting everything into spacetime, so that it can name and handle events. General relativity was a great change in this sense. It made spacetime alive; but, although dynamical, the relations between different events were still sharply defined. Quantum mechanics changed this, too. In such a sharply defined frame, objects became fuzzy; exact locations were substituted by probabilities of finding an object in a given region of space at a given instant of time.

The merging of quantum mechanics and special relativity gives rise to the well known difficulties of dealing with one-particle theories. Heisenberg's uncertainty principle and the finiteness of the speed of light, when put together, lead to creation and anihilation of particles if one tries to know what is going on in a region within the Compton wavelength of the 
particle under study [目]. The position-momentum uncertainty principle $\Delta x \Delta p \gtrsim 1$ can be written in terms of $x$, the position of a particle, and $E$, its energy, as $\Delta x \Delta E \gtrsim 1$ for relativistic particles (for which $E \sim p$ ). Therefore, if the position uncertainty is smaller than a Compton wavelength, i.e. $\Delta x \lesssim E^{-1}$, we get $E^{-1} \Delta E \gtrsim 1$ (throughout this work we set $\hbar=c=1$, so that the only dimensional constant is Planck's length $l_{*}=\sqrt{\mathrm{G}}$; also, irrelevant numerical factors are ignored). Then, the uncertainty in the energy of a particle is larger than its rest mass and this makes the concept of particle unclear [3]. We are facing a resolution limit, a minimum length for relativistic quantum mechanics: it is not possible to localize a particle with a better accuracy than its Compton wavelength. In this case, it means that a multiparticle theory is needed, i.e. quantum field theory. These problems are also reflected when one tries to define a position operator. In fact, an essentially unique position operator was defined by Newton and Wigner [4. But this operator, whose eigenvalues give the positions of a certain particle, has the following property: if a particle is localized in a certain region at a certain time, then at any arbitrarily close instant of time there is a non-zero probability of finding it anywhere; therefore, the particle would be travelling faster than light. This paradox disappears when, instead of dealing with a single particle (only positive frequency modes are considered), one allows the possibility of particle creation and anihilation (i.e. negative frequency modes enter the theory).

The next step, from the theoretical point of view, and also in the closely related energy scale, is to introduce gravity. Now spacetime is dynamical. It is affected by, and also affects, the objects and particles that it contains and that define it. A quantum uncertainty in the position of a particle implies an uncertainty in its momentum and therefore, due to the gravity-energy interaction, also implies an uncertainty in the geometry, which in turn introduces an additional uncertainty in position of the particle. Then, the expression for the position uncertainty will contain (at least in some approximation scheme) two pieces: the first one will correspond to the standard Heisenberg principle and the second one to the response of spacetime to the presence of this quantum uncertainty. The geometry is now subject to quantum fluctuations. What is the scale of these fluctuations? [5] To answer this question assume that we want to resolve a spherical region of radius $l$. We need a photon of wavelength smaller than $l$; its energy will be greater than $1 / l$ and therefore we will be putting an energy density $\rho$ greater than $1 / l^{4}$. Einstein's equations tell us that $\partial^{2} g \sim l_{*}^{2} \rho \gtrsim l_{*}^{2} / l^{4}$, where $l_{*}$ is Planck's length so that the gravitational potential (the spacetime metric) generated by this photon is $g \gtrsim l_{*}^{2} / l^{2}$ and therefore the length that is being measured will have an uncertainty $\sqrt{g l^{2}} \gtrsim l_{*}$. Thus, independently of any particular way of measuring the position, the distance between two events will have a minimum uncertainty of the order of Planck's length. This conclusion, which has been reached by means of rather crude arguments, can be obtained from a variety of more sophisticated thought-experiments and theoretical analyses of quantum gravity.

The existence of this fundamental length at Planck's scale beyond which the very concepts of space and time lose their meaning may have a similar meaning to the speed limit defined by the speed of light in special relativity, as pointed out by Markov [6, 7] (as quoted in Ref. [8]): there is no way of going beyond this border and its existence may be inferred through the relativistic corrections to the Newtonian theory. This would mean that a quantum theory of gravity could be constructed only in "this side of Planck's border". In fact, as we will see below, this analogy between quantum gravity and special relativity is quite 
close: in the latter you can accelerate forever even though you will never reach the speed of light; in the former, given a coordinate frame, you can reduce the coordinate distance between two events as much as you want even though the proper distance between them will not decrease beyond Planck's length.

The purpose of this paper is to review several scenarios in which a minimum length arises in the context of quantum gravity and also discuss some of the consequences. In what follows, we will be concerned with uncertainties due to the quantum fluctuations of the gravitational field itself or, in other words, the fluctuations that are introduced when a measurement is performed. However, there is another source of uncertainty that will not be considered here: the quantum fluctuations of the gravitational field due to the Heisenberg uncertainty relations that affect the source of the gravitational field [9, 10,11]. This uncertainty decreases with distance to the source and it vanishes for sufficiently large distances.

\section{MINIMUM LENGTH}

A minimum length at Planck's scale can be obtained from the study of some thoughtexperiments and approaches to quantum gravity. We describe some of them in this section.

\section{A. Microscope thought-experiments}

The process of detecting a particle with a microscope and some related thoughtexperiments were carefully analyzed by Mead [12]. He gave a variety of treatments from the simplest in which gravity was regarded as Newtonian, to the most sophisticated in which general relativity was fully implemented. For simplicity, we will reproduce the Newtonian argument.

Apart from the uncertainty due to the lack of knowledge of the exact direction of the photon scattered by the observed particle, $\Delta x \gtrsim 1 / \Delta p$, there is another piece that contributes to this uncertainty. This is due to the gravitational interaction between the observed particle and the photon. The particle will be attracted towards the photon with an acceleration given by the Newtonian gravitational field $l_{*}^{2} \omega / r^{2}$, where $\omega$ is the energy of the photon and $r$ is the radius of the region of strong interaction (i.e. during the time $r$ that the photon is within this region, it cannot be focussed). During this time, the particle acquires a velocity $l_{*}^{2} \omega / r$ and therefore travels a distance $l_{*}^{2} \omega$ in the direction in which the photon is moving, which is unknown. Projecting down to the $x$-axis, we find $\Delta x \gtrsim l_{*}^{2} \Delta p$. Combining both pieces of uncertainty, we get

$$
\Delta x \gtrsim \max \left(\frac{1}{\Delta p}, l_{*}^{2} \Delta p\right) \gtrsim l_{*} .
$$

The physical explanation seems evident from this expression. To get a high resolution you need high energy photons (due to the standard Heisenberg's uncertainty relation), but the higher the energy of the photons, the higher the gravitational interaction and therefore the higher the disturbance. If the energy is too high, gravitational effects will seriously affect the particle and spoil the advantages of using high energy photons. 
Mead also showed that there is a limit in the time resolution when synchronizing clocks (see also Ref. [13]). We will make use of the full general relativity theory (rather than the Newtonian limit). In fact, the argument given above, although qualitatively correct, is not valid since the Newtonian approximation breaks down at the scales that we are working. Assume that we want to synchronize a clock with another standard clock. We will do this by interchanging photons. The reading of our clock has at least two sources of uncertainty. The first one is due to the Heisenberg time-energy uncertainty relation, which constrains the accuracy in the emission or absorption of the photon, and therefore the reading of our clock, to be smaller than $1 / \Delta \omega$. The second source of uncertainty is the gravitational interaction between the photon and the clock. Assume that the clock and the photon strongly interact in a region of radius $r$, and therefore during a period $r$ (provided that the clock remains stationary). The duration of this interaction, according to the clock, will be $\sqrt{g_{00}} r$, where $g_{00}$ is the time-time component of the gravitational field generated by the photon (and suffered by the clock) and that can be seen to have the form

$$
g_{00}=1-\frac{4 l_{*}^{2} \omega}{r} .
$$

The uncertainty in the duration of the interaction due to the frequency spread $\Delta \omega$ is

$$
\frac{2 l_{*}^{2} \Delta \omega}{\sqrt{1-4 l_{*}^{2} \omega / r}} \gtrsim 2 l_{*}^{2} \Delta \omega .
$$

The uncertainty in the reading of our clock will then be greater than both uncertainties:

$$
\Delta t \gtrsim \max \left(\frac{1}{\Delta \omega}, l_{*}^{2} \Delta \omega\right) \gtrsim l_{*} .
$$

It should be mentioned that these uncertainty relations are low-energy approximations to what the full theory of quantum gravity would give. Indeed, they are made up of two pieces: the first one is due to the Heisenberg uncertainty principle and the second is due to the dynamical response of spacetime to such uncertainty. But this alteration should induce a new uncertainty in the gravitational field, which would again add an additional uncertainty, and so on. This whole series of terms is an expression of the nonlinearity of the gravitational interaction or, in other words, of the equivalence principle.

\section{B. Path-integral quantum gravity}

From different points of view, both Padmanabhan [14,15, 16, 17] and Greensite [18] inferred the existence of a minimum length within a path integral formulation of quantum gravity; namely, the former worked in a theory in which the conformal factor is quantized [19] and the latter in lattice quantum gravity (see also Refs. [20,21,22]). They both reached the result that the coincidence limit, in which the difference between coordinates of two events in a given frame or the separation between lattice points goes to zero, of the proper interval between them is not zero but Planck's length. However, there are some discrepancies as to the origin of this residual length as we will see. Let us review their arguments, beginning with Padmanabhan's approach. 
For the sake of simplicity, we will restrict our attention to the case of conformally flat metrics

$$
g_{\mu \nu}(x)=\left[1+\phi(x)^{2}\right] \eta_{\mu \nu},
$$

although the same result is obtained if we remove this restriction. The path integral over the conformal fluctuation $\phi$

$$
\int \mathcal{D} \phi \exp \left(\frac{-i}{l_{*}^{2}} \int d^{4} x \eta^{\mu \nu} \nabla_{\mu} \phi \nabla_{\nu} \phi\right)
$$

can be evaluated in a closed form. This allowed Padmanabhan to write the probability amplitude for a measurement of the conformal fluctuation to give a value $\phi$ :

$$
\mathcal{A}(\phi)=\left(\frac{l}{l_{*}}\right)^{1 / 4} \exp \left(-\frac{l^{2}}{l_{*}^{2}} \phi^{2}\right),
$$

where $l$ is the resolution of the measuring apparatus (the radius of the region over which it averages). It should be noted that if the region over which one measures is very large $\left(l \gg l_{*}\right)$, then the probability amplitude $\mathcal{A}$ will have a sharp peak at flat spacetime (i.e. $\phi=0)$. This distribution provides a kind of uncertainty relation

$$
\Delta \phi l \gtrsim l_{*} .
$$

The greater the resolution (i.e. the smaller $l$ ), the greater the fluctuations of the conformal factor will be.

Let us now calculate the coincidence limit for two close events of the vacuum expectation value of its proper interval:

$$
\lim _{x \rightarrow y}\left\langle l^{2}(x, y)\right\rangle=\lim _{x \rightarrow y}[1+\langle\phi(x) \phi(y)\rangle] l_{0}^{2}(x, y)
$$

where $l_{0}^{2}(x, y)$ is the proper interval in the metric $\eta_{\mu \nu}$. However, from the form of the action for the field $\phi$ (it is that of a free scalar field with the "wrong sign") it is straightforward to see that

$$
\langle\phi(x) \phi(y)\rangle=\frac{l_{*}^{2}}{l_{0}^{2}(x, y)}
$$

so that $l_{*}$, Planck's length, is the minimum proper interval for any two events.

Greensite [18], on the other hand, considered linearized gravity in a cubic lattice of points that represents spacetime. Again, the arguments can be extended to full general relativity without any change in the final result. Also, the tetrad formulation is chosen in order to prevent configurations with $\operatorname{det}(-g)<0$ from giving any contribution to the path integral. Then the gravitational field is represented by the tetrad

$$
e_{\mu}^{a}=\delta_{\mu}^{a}+l_{*}^{2} b_{\mu}^{a}
$$

and the path integration is performed over the field $b_{\mu}^{a}$. The proper distance separation between two neighbouring events in the lattice (in the $x$-direction, for instance) is 


$$
\left\langle d s^{2}\right\rangle=\left[1+l_{*}^{2} \delta_{a b}\left\langle b_{1}^{a}(x) b_{1}^{b}(x)\right\rangle\right] \epsilon^{2},
$$

where $\epsilon$ is the lattice parameter. As above,

$$
\delta_{a b}\left\langle b_{1}^{a}(x) b_{1}^{b}(x)\right\rangle \sim \epsilon^{2}
$$

and therefore,

$$
\lim _{\epsilon \rightarrow 0} \sqrt{\left\langle d s^{2}\right\rangle} \sim l_{*}
$$

As Greensite pointed out, in both approaches the fact that configurations with negative $\operatorname{det}(-g)$ are precluded shifts the expectation value of $g_{\mu \nu}$, although the origin of this shift is different: fluctuations of the conformal factor in Padmanabhan's approach and all components of the metric in Greensite's (in the latter the conformal fluctuations tend to depress this effect rather than enhance it). There is also another difference between both approaches. In the lattice, the physical separation between two points separated by a finite coordinate distance is infinite in the $\epsilon \rightarrow 0$ limit, while in Padmanabhan's approach, it is finite and regarded as a correction.

\section{String theory}

String theory already contains a minimum length as can be seen from a simple argument 23,24]: After one fixes the reparametrization gauge, the partition function for a string can be written in the discretized form

$$
Z=\int \mathcal{D} x \exp \left\{i \frac{\text { const. }}{l_{*}^{2}} \sum \epsilon^{2}\left[\left(\frac{\delta_{\tau} x}{\epsilon}\right)^{2}+\left(\frac{\delta_{\sigma} x}{\epsilon}\right)^{2}\right]\right\},
$$

where the constant appearing in the exponent depends on the particular model that one is considering. We can see that, due to the fact that the worldsheet is two dimensional, the $\epsilon$-factors cancel out, thus leading to an $\epsilon$-independent spacetime distance

$$
\left\langle(\delta x)^{2}\right\rangle \sim \text { const. } l_{*}^{2}
$$

Through a detailed analysis of thought-experiments involving high energy string collisions 25,26], and also through a renormalization group type analysis in string theory [24,27,28] it was found that this result could be qualitatively written in the form (see, for instance, Refs. [29,30|)

$$
\Delta x \gtrsim \frac{1}{\Delta p}+\text { const. } l_{*}^{2} \Delta p
$$

essentially the same expression that had been previously obtained in microscope thoughtexperiments. The linear term is due to the behaviour of strings at high energy: They spread out, their size being proportional to their energy.

Duality is also involved in the lower bound to the spacetime distance [31, 32, 33, 34, 35, 36]. Since it is not possible to distinguish a compactification of radius $r$ from another of radius 
$l_{*}^{2} / r$, very small distances are the same as very large distances and thus Planck's length represents an insurmountable border (see, for instance, Ref. [30]).

On the other hand, Klebanov and Susskind [37] have shown that one can obtain an exact description of the bosonic string theory by constructing a discrete field theory. Therefore, the number of short-distance degrees of freedom of the bosonic string must be much smaller than in any conventional field theory. This may be one of the reasons for the exponential damping that high-energy fixed-angle scattering amplitudes suffer [38,39], and for the slower growth of thermal partition functions at high temperature [40]. These are indications of the impossibility of resolving the short-distance structure of string theory.

\section{Loop representation}

In the loop representation [41,42,43] (for a review, see for instance Ref. [44]) of nonperturbative canonical quantum gravity, geometry is studied non-perturbatively, so that background fields do not play any role. Operators that carry the metric information, but that are made finite without the aid of any background structure, can be defined. These operators can be used to define loop states that, in the classical limit, provide a classical geometry. It is remarkable that some of these finite operators have discrete spectrum, i.e. a discrete small-distance structure. One such operator is the area operator.

Let us consider a surface defined by a physical reference system 455. A matter field can play such role, the surface being the set of points where the field takes a given value. Then, the area of this surface is quantized, i.e. it is an integer multiple of the elementary Planck's area [46,47]. The integer number can be seen to be the number of times that the loops cross the surface, so that each loop contributes a unit (Planck's area) to the total area when it crosses the surface, regardless of the orientation of the intersection.

One should note a difference between this approach and any other in this work. Here, a small-distance scenario is built and the consequences of this scenario are the recovery of a classical geometry in the limit of large distances and the realization that the small-scale structure is discrete. On the other hand, the other analyses that we have studied here approach the lower bound to the distance uncertainty from above, that is from semiclassical reasonings concerning the quantum fluctuations of the gravitational field.

\section{E. Black hole horizon}

It has been proposed by Bekenstein [48 that the area of a Kerr black hole should be quantized (see also Refs. 449,50,51]). For simplicity, we will concentrate on a Schwarzschild black hole. He argued that the area of a black hole has a close resemblance to the action integral $\oint p d x$ of a periodic mechanical system: first, both of them are adiabatic invariants; and second, both of them have dimensions of an action. Therefore, he proceeded by analogy and stated that the area of a black hole should obey the equivalent to the Bohr-Sommerfeld quantization rule:

$$
A=\frac{1}{2} l_{*}^{2} n,
$$


where $\mathrm{n}$ is an integer and the coefficient $1 / 2$ can be obtained by means of the correspondence principle: for large $n$ the system must have a classical behaviour. This result has been reproduced from the semiclassical analysis of the Wheeler-DeWitt equation for a collapsing dust star [52]. Bekenstein also realized that, except for the ground state $n=1$, the Compton radius of the black hole $l_{*}^{2} / \sqrt{A} \sim l_{*} / n$ is always smaller than its gravitational radius $\sqrt{A} \sim$ $l_{*} \sqrt{n}$. Only for a black hole in its quantum state will the two radii coincide, and be equal to Planck's length.

Quite recently, Maggiore [53] has found that an uncertainty relation that gives rise to a minimum length can also be derived from the measurement of the radius of a black hole. He realized that in classical general relativity the observer does not have access to the horizon and the only way of measuring its radius is through the relation between the radius of the apparent horizon and the parameters of the black hole (mass, charge, etc.). Therefore, this relation must be regarded as a definition rather than as an experimentally testable relation. The situation changes when quantum gravity is put into the theory. Hawking radiation allows one to make an independent, direct measurement of the area of the black hole and, consequently, the relation between the radius of the apparent horizon and the black hole parameters can be experimentally tested (at least in thought experiments).

Let us perform one of these thought experiments by dropping a photon of frequency $\omega$ into an extremal black hole. Then the mass of the black hole will be incremented by an amount $\omega$ and will decay to the extremal stable state. Assume that this decay is accompanied by an emission of a single photon of frequency $\omega$ (if more photons are emitted the same conclussion will be reached). Note that this assumption is plausible since the emission produced by a nearly extremal black hole need not be thermal [54]. By detecting this photon and repeating the measurement many times, one can "see" the black hole. There is an uncertainty due to the standard Heisenberg principle: $\Delta x \geq 1 / \Delta p$ where $\Delta p$ represents the uncertainty in the momentum of the black hole, due to the emission of the photon, whose direction is not well known. But also, since the mass of the black hole will pass from $M+\omega$ to $M$ during the measurement, we can only know the value of its mass with an uncertainty $\omega$. Accordingly, the radius of the apparent horizon will have an uncertainty $\Delta x \gtrsim l_{*}^{2} \omega \gtrsim l_{*}^{2} \Delta p$, so that the uncertainty in the size of the hole is given by the already familiar expression

$$
\Delta x \gtrsim \max \left(\frac{1}{\Delta p}, l_{*}^{2} \Delta p\right) .
$$

\section{ULTRAVIOLET CONVERGENCE}

The lower bound on the proper distance between any two events may become the ultimate ultraviolet regulator [55,56], since any distance below this bound loses its meaning. Let us illustrate this issue in the case of a massless scalar field in curved spacetime [55, 14] (see also Ref. [57]). The Green function evaluated in a classical spacetime has the well-known form

$$
D(x, y) \sim \frac{1}{l_{0}^{2}(x, y)+i \epsilon}
$$


which is divergent, i.e. goes to infinity for small proper distances. However, when quantum fluctuations of spacetime are taken into account, the notion of distance is no longer unique. Therefore, an average over all possible distances provided by the quantum fluctuations must be performed. This amounts to replace the propagator $D(x, y)$ by its expectation value

$$
\langle D(x, y)\rangle \sim \frac{1}{l^{2}(x, y)+i \epsilon} \sim \frac{1}{l_{0}^{2}(x, y)+l_{*}^{2}+i \epsilon} .
$$

We see that now the ultraviolet limit $x \rightarrow y$ gives a finite propagator rather than a divergent one, because of the existence of a minimum proper interval, which acts as a high-energy cutoff. Indeed, by Fourier-transforming this expression (and rotating it to Euclidean space) we obtain the Euclidean propagator in momentum space

$$
\langle D(k)\rangle \sim \frac{l_{*}}{|k|} \mathrm{K}_{1}\left(l_{*}|k|\right) .
$$

By asymptotically expanding the modified Bessel function $\mathrm{K}_{1}(|k|)$, we can see how the presence of Planck's length affects the propagator

$$
\langle D(k)\rangle \sim\left\{\begin{array}{ll}
1 / k^{2} & \text { when }|k| \rightarrow 0 \\
\left(l_{*} /|k|^{3}\right)^{1 / 2} e^{-l_{*}|k|} & \text { when }|k| \rightarrow \infty
\end{array} .\right.
$$

It causes a strong damping on the high-energy contributions to the propagator.

As expected, once the propagator has been made finite, one-loop calculations show [14] that the effective potential is also finite.

\section{KINEMATICAL ORIGIN OF THE UNCERTAINTY RELATIONS}

In quantum mechanics, the uncertainty relations are not dynamical. They come from the kinematical structure of the theory, i.e. the fact that position and momentum operators do not commute gives rise to an uncertainty relation between them. The same will happen with any two observables that do not commute: it will not be possible to measure them simultaneously. Now we ask the question of whether the generalized uncertainty relations can also be derived from the kinematical structure of the theory. From the examples and procedures that we have discussed, it seems quite clear that the two pieces that contribute to the uncertainty in the position are very different in nature. One of them clearly has a kinematical structure, stating that objects are wavelike. This part does not contain any dimensional parameter or coupling constant. On the other hand, the second term involves gravitation, which is a dynamical theory of the interaction between matter and spacetime. That it contains a dimensional constant, Planck's length, is a reflection of this fact. Therefore, one can ask how it is possible to make such dynamically generated uncertainty relations a consequence of some kinematical structure. The answer relies on the fact that even though one could find it, it would be necessary to give the dynamics of the system in order to provide it with a clear well-defined meaning, since a suitable non-local change of variables can make the uncertainty relation look like Heisenberg's. However, this non-local change of variables will turn the action non-local and non-local actions contain a minimum length in them [23. 
In view of this discussion, one cannot truly say that the generalized uncertainty relations are due to kinematics exclusively, as is the case with Heisenberg's principle. This discussion of the kinematical or dynamical origin of the generalized uncertainty relations may well be relevant to the final full quantization of gravity since, as mentioned in the introduction, this seems to be the origin of many problems when one is quantizing gravity: the active response of spacetime to quantum fluctuations.

An early attempt to give a kinematical meaning to the generalized uncertainty relations was made by Mead [58] (see also Ref. [59]). His idea was to represent a physical quantity $A$ such that $\Delta A \geq \alpha$ by an operator that fails to commute with some Hermitian bounded operator $Q$. Indeed, if $-1 \leq Q \leq 1$ and $[A, Q]=i \alpha$, for instance, then $\Delta A \Delta Q \geq \alpha$; since $\Delta Q \leq 1$, we see that $A$ will satisfy the required relation $\Delta A \geq \alpha$. Such an operator $A$ will be called "indeterminate", following Mead. In ordinary quantum mechanics, the momentum of a particle in a box is an indeterminate operator, for example. Of course, in this analysis, due care must be taken with boundary conditions and domains of the operators. The operator $Q$ associated to the indeterminate position operator in quantum gravity will contain information about the origin and the physical significance of the fundamental length, i.e. about the quantum theory of gravity.

By considering gravity as the gauge theory of the deSitter group, Townsend 60 introduced Planck's length into gravity kinematically. The appearance of this dimensional constant is due to the non-commutativity of the generators of translations. Quantum gravity effects become relevant at the same scale at which this non-commutativity is important. Recently, Maggiore [61, 62 (see also Refs. 63, 64]) has pushed this kind of arguments further and has found an algebra that gives rise to the generalized uncertainty relations and that, under quite general assumptions, is essentially unique. This algebra turns out to be a deformed Heisenberg algebra

$$
\left[x_{i}, x_{j}\right]=-i l_{*}^{2} \epsilon_{i j k} J_{k}, \quad\left[x_{i}, p_{j}\right]=i \delta_{i j}\left(1+l_{*}^{2} E^{2}\right)^{1 / 2},
$$

where $J_{k}$ are the generators of the three-dimensional rotations and $E$ is the energy of the particle, $E^{2}=p^{2}+m^{2}$. This result can also be obtained by considering the deformed Poincaré algebra, where position operators are substituted by suitably deformed NewtonWigner operators [61], rather than the undeformed one.

There is an important difference between Maggiore's approach to the existence of a minimum length and the other approaches that we have considered so far. It is the issue of whether this resolution limit also affects the time variable or not. In view of the deformed Heisenberg algebra proposed by Maggiore, only spatial separations will be subject to generalized uncertainty relations. However, as we have seen in the approaches above, the generalized uncertainty relations should apply to all position and time measurements, the minimum uncertainty being intrinsic also to synchronization of clocks. What there seems to be subject to minimum uncertainty is the proper spacetime interval rather than the spatial separations alone. 


\section{MEASURING THE GRAVITATIONAL FIELD}

\section{A. Classical measurement devices}

The measurement process of the gravitational fields naturally leads to a minimum volume of the measurement domain. Based on the work by Bohr and Rosenfeld 65, 66, 67,68 (see e.g. Ref. [69]) for the electromagnetic field, Peres and Rosen [70 and then DeWitt 771] carefully analyzed the measurement of the gravitational field and the possible sources of uncertainty (see also Refs. 72, 73, 74]). Their analysis was carried out in the weak-field approximation (the magnitude of the Riemann tensor remains small for any finite domain) although the features under study can be seen to have more fundamental significance. This approximation imposes a limitation on the bodies that generate and suffer the gravitational field: their masses must be small when compared with their linear dimensions. It is worth noting that this minimum length does not appear in the case of an electromagnetic field. The main reason for this is that in this case, the relevant quantity that is involved in the uncertainty relations is the ratio between the charge and the mass of the test body, and this quantity can be made arbitrarily small. This is certainly not the case for gravitational interactions, since the equivalence principle precisely fixes the corresponding ratio between gravitational mass and inertial mass, and therefore it is not possible to make it arbitrarily small. Let us go into more detail in the comparison between the electromagnetic and the gravitational fields as far as uncertainties in the measurement are concerned.

The arguments of Bohr and Rosenfeld lead to the uncertainty relation

$$
\Delta F l^{3} \gtrsim \frac{q}{m},
$$

where $F$ is the electromagnetic field strength and $l, q$ and $m$ are the linear dimension, the charge and the mass of the test body, respectively, provided that the test body satisfies the conditions

$$
l \gtrsim \frac{1}{m}, \quad l \gtrsim \frac{q^{2}}{m} .
$$

They are the reflection of the following assumptions concerning the test body: the measurement of the field average over a spacetime region, whose linear dimensions and time duration are determined by $l$, is performed by determining the initial and final momentum of a uniformly charged test body; the time interval required for the momentum measurement is small compared to $l$; any back-reaction can be neglected if the mass of the test body is sufficiently high; and finally, the borders of the test body are separated by a spacelike interval. These conditions can be summarized by saying that the test body must be classical from both the quantum and the relativistic point of view. We see from the uncertainty relation for the electromagnetic field that an infinite accuracy can be achieved if an appropriate test body is used. This is not the case for the gravitational interaction. Indeed, the role of $F$ is now played by $\Gamma / l_{*}$, where $\Gamma$ is the connection, and the role of $q$ is played by $l_{*} m$. It is worth noting [70], as mentioned above, that by virtue of the equivalence principle, active gravitational mass, passive gravitational mass and energy (rest mass in the Newtonian limit) are all 
equal, and hence, for the gravitational interaction, the ratio $q / m$ is the universal constant $l_{*}$. The two requirements of Bohr and Rosenfeld are now

$$
l \gtrsim 1 / m, \quad l \gtrsim l_{*}^{2} m,
$$

so that $l \gtrsim l_{*}$. This means that the test body should not be a black hole, i.e. its size should not exceed its gravitational radius, and that both its mass and linear dimensions should be larger than Planck's mass and length, respectively. As in the electromagnetic case, Bohr and Rosenfeld requirements can be simply stated as follows: the test body must behave classically from the points of view of quantum mechanics, special relativity and gravitation. Otherwise, the interactions between the test body and the object under study would make this distinction (the test body on the one hand and the system under study on the other) unclear as happens in ordinary quantum mechanics: the measurement device must be classical or it it is useless as a measuring apparatus. In this sense, in the context of quantum gravity, Planck's scale stablishes the border between the measuring device and the system that is being measured. Then, the uncertainty relation for the connection can be written as

$$
\Delta \Gamma l^{3} \gtrsim l_{*}^{2}
$$

or in terms of the metric tensor,

$$
\Delta g l^{2} \gtrsim l_{*}^{2}
$$

The left hand side of this relation can be interpreted as the uncertainty in the proper separation between the borders of the region that we are measuring, so that it again states the minimum position uncertainty relation. It is worth noting that it is the concurrence of the three fundamental constants of nature $\hbar, c$, and $\mathrm{G}$ that leads to a resolution limit. If any of them is dropped then this resolution limit disappears.

To end this section, a brief comment on the meaning of these uncertainty relations is in order. DeWitt [71] and Peres and Rosen [70] interpreted them as providing a lower bound to the size of the measurement domain and an indication of the necessity of quantizing the gravitational field. On the other hand, Rosenfeld [66] and Borzeszkowski and Treder [8] regarded these uncertainties as an expression of the impossibility of measuring quantum gravity effects, comparing this situation with the controversy that the quantization of the electromagnetic field raised in the early thirties [75,65].

\section{B. Continuous measurement}

Assume that we continuously measure an observable $Q$, within the framework of ordinary quantum mechanics. Let us call $\Delta q$ the uncertainty of our measurement device. This means that, as a result of our measurement, we will obtain an output $\alpha$ that will consist of the result $q(t)$ and any other within the range $(q-\Delta q, q+\Delta q)$. The probability amplitude for an output $\alpha$ can be written in terms of path integrals [76]:

$$
A[\alpha]=\int_{\alpha} \mathcal{D} x e^{i S},
$$


where $\alpha$ denotes not only the output but also the set of trajectories in configuration space that lead to it. For a given uncertainty $\Delta q$, the set $\alpha$ is fully characterized by its central value $q$. We are particularly interested in studying the shape of the probability amplitude A. More precisely, we will pay special attention to its width $\Delta \alpha$ [76, 77] (for a decoherent histories approach see Ref. [78]).

There are two different regimes of measurement, classical and quantum, depending on whether the uncertainty of the measuring device is large or small. If we define the set of classical trajectories $\mathcal{C}$ as

$$
\mathcal{C}=\left\{x /\left|S(x)-S\left(x_{c}\right)\right| \lesssim 1\right\}
$$

then the classical regime of measurement will be accomplished if $\Delta q$ is large enough so that $\mathcal{C} \subset \alpha_{c}$, where $\alpha_{c}$ is the set of trajectories that lie in the corridor defined by $q_{c}-\Delta q$ and $q_{c}+\Delta q$. Since the set $\mathcal{C}$ is the one that gives significant contribution to the path integral (the interference is constructive), both the $\alpha_{c}$-restricted and the unrestricted path integral will give about the same result. In this regime, the width of the probability amplitude $\Delta \alpha$ can be seen to be proportional to the uncertainty $\Delta q$. Indeed, the greater $\Delta q$, the greater will be the number of sets $\alpha$ that contain the set of classical trajectories $\mathcal{C}$. Also, the uncertainty on the action can be estimated to be

$$
\Delta S=\left|S\left(q_{c}+\Delta \alpha\right)-S\left(q_{c}\right)\right| \gtrsim\left|S\left(q_{c}+\Delta q\right)-S\left(q_{c}\right)\right| \gtrsim 1
$$

since $\Delta \alpha \gtrsim \Delta q$.

The quantum regime of measurement occurs when $\Delta q$ is so small that no set $\alpha$ contains the set of classical trajectories. Rather, it will happen that $\mathcal{C} \subset \alpha_{c}$. Now the width of the probability amplitude is $\Delta \alpha \sim 1 / \Delta q$. Indeed, the sets $\alpha$ that give a significant contribution to the path integral are those that satisfy $|S(q+\Delta q)-S(q)| \lesssim 1$. Therefore, the smaller $\Delta q$ is, the easier will be this inequality to be satisfied. The uncertainty in the action will be greater than unity, because at least all the sets $\alpha$ contained in $\mathcal{C}$ will have a high probability amplitude.

To summarize, in any regime of measurement, the action uncertainty will be greater than unity. In view of the discussion above, the width $\Delta \alpha$ of the probability amplitude will achieve its minimum value, i.e. the measurement will be optimized, for uncertainties in the measurement device $\Delta q$ that are neither too large nor too small. When this minimum non-vanishing value is achieved, the uncertainty in the action is also minimized and set equal to one. The limitation on the accuracy of any continuous measurement is, of course, an expression of Heisenberg's uncertainty principle. Since we are talking about measuring trajectories in some sense, a resolution limit should appear, expressing the fact that position and momentum cannot be measured simultaneously with infinite accuracy. In the classical regime of measurement, the accuracy is limited by the intrinsic uncertainty of the measuring device. On the other hand, when very accurate devices are employed, quantum fluctuations of the measuring apparatus affect the measured system and the final accuracy is also affected. The maximum accuracy is obtained when there is achieved a compromise between keeping the classical uncertainty low and keeping quantum fluctuations also small.

This discussion is quite similar to the arguments that we have made throughout this work concerning the existence of a minimum length, a resolution limit in the context of quantum 
gravity. In fact, any measurement of the gravitational field is not only extended in time, but also extended in space. These measurements are made by determining the change in the momentum of a test body of a given size. That measurements of the gravitational field have to be extended in spacetime, i.e. they have to be continuous, is due to the dynamical nature of this field.

Let us consider [77 a measurement of the scalar curvature averaged over a spacetime region of linear dimension $l$, given by the resolution of the measuring device (the test body). The action is

$$
S=\frac{1}{l_{*}^{2}} \int d^{4} x R \sqrt{-g}
$$

where the integral is extended over the spacetime region under consideration, so that it can be written as

$$
S=\frac{1}{l_{*}^{2}} \bar{R} l^{4}
$$

$\bar{R}$ being the average curvature. The action uncertainty principle $\Delta S \gtrsim 1$ gives the uncertainty relation for the curvature

$$
\Delta \bar{R} l^{4} \gtrsim l_{*}^{2}
$$

equivalent to the previously obtained relations for the connection and the metric.

We can see that the problem of measuring the gravitational field, i.e. the structure of spacetime, can be traced back to the fact that any such measurement is non-local, i.e. the measurement device is aware of what is happening at different points of spacetime and takes them into account. In other words, the measurement device averages over a spacetime region. The equivalence principle also plays a fundamental role: the measurement device cannot decouple from the measured system and back reaction is unavoidable.

\section{SMALL-DISTANCE STRUCTURE}

\section{A. Saturation of Lorentz transformations}

We have seen that quantum, special relativistic and gravitational effects, all together, give rise to a fundamental length. Also, they may cause Lorentz transformations to saturate at the Planck's scale. Indeed, let us apply a Lorentz transformation characterized by the boost parameter $\gamma$ to a particle of rest-mass $m$ and linear dimension $l$. For the laboratory system of reference, its linear dimension will be $l / \gamma$, its mass $\gamma m$, its Compton wavelength $1 / \gamma m$ and its gravitational radius $l_{*}^{2} \gamma m$. Its size will be given by the largest of these quantities. If the boost is smaller than the critical value

$$
\gamma_{c}=\frac{1}{l_{*}} \max (1 / m, \sqrt{l / m}),
$$

then the size of the particle will be determined by either its linear dimension or its wavelength, whichever is larger, and it will decrease with $1 / \gamma$. Once the boost parameter reaches 
its critical value, the gravitational radius will define the size of the particle (assuming the absence of other effects that would contribute to an increasing of its size, such as those considered below) and increase as $l_{*}^{2} \gamma m$. Therefore, the particle will have a minimum size no matter how large the boost is. Although the critical value, $\gamma_{c}$, of the parameter $\gamma$ for the saturated boost depends on the mass and linear dimension of the particle, the minimum size that it may ever reach is Planck's length, independent of the nature of the particle. Of course, this could be expected because if Lorentz transformations did not saturate, then highly boosted particles could be used as arbitrary accurate probes, in contradiction with the minimum length uncertainty relation.

Due to the existence of a minimum proper interval, the notion of event loses one of its characteristic features: it is no longer an invariant concept [79,80,81]. The uncertainty in the radius of the black hole horizon may be interpreted as giving rise to a membrane, a stretched horizon, from the point of view of an observer at rest with respect to the black hole (for a study of the effect of a Planck's length cutoff on black-hole evaporation, see also Ref. [82]). However, this membrane would be real only for this observer at rest (its reality would be dramatically shown if this observer were too close to the membrane since its extremely high temperature would burn him to ashes). A free-falling observer would not see or feel this membrane since, for him, nothing special would take place at the horizon; in fact, there would be no horizon for him. The absolutely different perceptions of both observers can be stated [83,84] (see also Refs. 85,86] for related ideas) in terms of a black hole complementarity principle: events are observer-dependent, in the same manner as in quantum mechanics the answer to the question "Is an electron particlelike or wavelike?" depends on the kind of measure that is performed, or as in special relativity the issue of simultaneity of two events depends on the observer. The physical laws appear the same in all reference systems but the description of the physical reality may vary from observer to observer.

From the black hole complementarity principle, one can also derive the saturation of Lorentz contraction when a particle that is falling to a black hole acquires an energy close to Planck's scale (as it reaches the stretched horizon). This saturation results also in a minimum size for the particle but of a slightly different nature from the one discussed above. Here the notion of size that is involved is that of "size occupied by information", which, as discussed by Susskind [80], may not coincide with the wavelength or the gravitational radius of the particle. According to the black hole complementarity principle, the stretched horizon must thermalize and spread throughout the information that falls to the black hole, to be reemited afterwards. This means that, as the particle reaches the stretched horizon (at that stage the boost of the particle as seen by an observer at rest with respect to the black hole is extremely large, close to saturation), its longitudinal size must fully overlap with the stretched horizon radial size, which is of the order of Planck's length, and its transverse size must cover the whole black hole in order to transfer all the information to the stretched horizon homogeneously. Therefore, as the particle is boosted, two different, simultaneous processes occur: first, the longitudinal information size of the particle decreases up to Planck's length and, second, its transverse information size increases until it covers the stretched horizon. 


\section{B. Causality}

The light cone itself is affected by the fluctuations of the gravitational field. The uncertainty in the speed of photons (the slope of the light cone) will increase with the uncertainty in the gravitational field:

$$
\frac{\Delta v_{p h}}{v_{p h}} \sim \frac{\Delta g}{g} \gtrsim \frac{l_{*}^{2}}{g l^{2}}
$$

For fluctuations in the gravitational field of the order of the gravitational field itself or, in other words, when the size of the probe is close to Planck's scale, the uncertainty in the light cone slope is as large as the slope itself. This means that the distinction between spacelike and timelike separations is lost close to Planck's scale.

\section{Quantum cosmology}

In the quantum cosmology approach to quantum gravity (see e.g. Ref. 87]), wave functions are required to oscillate so that they represent classical spacetime when the radius of the universe is larger than Planck's length, i.e. they must have a classical behaviour. However, these wave functions are also well defined for small radii. For these values, at least in simple models, the wave function will represent a classically forbidden region (it will no longer oscillate). This is nicely illustrated by a simple minisuperspace model: de Sitter spacetime. We will consider a homogeneous and isotropic spacetime described by the Friedmann-Robertson-Walker metric

$$
d s^{2}=-d t^{2}+a(t)^{2} d \Omega_{3}^{2},
$$

where $a(t)$ is the scale factor and $d \Omega_{3}^{2}$ is the metric of the unit three-sphere. Then, the Wheeler-DeWitt equation (up to operator ordering ambiguities) can be written as

$$
\left(-\partial_{a}^{2}+a^{2}-\lambda a^{4}\right) \Psi(a)=0,
$$

where $\lambda$ is the dimensionless cosmological constant and $\Psi$ is the wave function of the universe. For small $a$, the potential term will be positive and therefore will represent a classically forbidden region (tunnelling region). In this simple example, the behaviour of the wave function is

$$
\Psi(a) \sim\left\{\begin{array}{l}
\exp \left\{ \pm \frac{1}{3 \lambda}\left(1-\lambda a^{2}\right)^{3 / 2}\right\} \quad \text { when } a \ll 1 / \sqrt{\lambda} \\
\exp \left\{ \pm \frac{i}{3 \lambda}\left(\lambda a^{2}-1\right)^{3 / 2}\right\} \text { when } a \gg 1 / \sqrt{\lambda}
\end{array} .\right.
$$

It is oscillatory for large radii (classical behaviour) and exponentially damped for small radii (classically forbidden configurations). Therefore, the small radius region will represent a genuine quantum spacetime, without a fixed metric, causality relations or any other classical structure. In our attempt of probing smaller and smaller distances, as the border that separates both regions is reached, the quantum fluctuations around the classical metric predicted by the oscillatory regime become large. In fact, it is close to this point where the semiclassical approximation breaks down. 


\section{Topology change}

It has been suggested that the quantum fluctuations of the metric would convert spacetime into a boiling magma in which topology change is continuously happening [5, 88, 89, 90 . This would mean that time evolution would no longer exist and causality would lose its meaning, as has been seen to be the case due to the light cone fluctuations. In view of these quantum fluctuations it is not surprising that quantum tunnelling effects may take place. They would cause particles to disappear from a region of spacetime and reappear in another one. These tunnelling effects could be seen as instantons, configurations in Euclidean space that would connect, quantum mechanically, different regions of spacetime otherwise disconnected or far apart from each other. They are the so-called wormholes 91] (for a review, see for instance Refs. 92,93,94]). They admit also a full quantum treatment in terms of wave functions $91,95,96,97$. The picture displayed above just corresponds to the semiclassical approximation. Actually, the (Euclidean) path integral approach considers any possible spacetime topology 91,98: the transition amplitude between any two threegeometries can be written as the sum over all possible topologies and over all possible metrics defined on them that match the boundary data. Among these topology changes, wormholes have deserved special attention. They provide a scenario in which the minimum length uncertainty relation can be pictorially realized: wormholes would delocalize any probe of sufficiently small size. This nonlocality of the Planck's scale dynamics would be reflected in the low-energy physics as effective interactions [91,99, 100,101 that would turn the coupling constants of the probe, its mass/size among others, into dynamical variables subject to a probability distribution 99. This would be the low-energy remnant of the Planck's scale uncertainties. Even more, it has been argued that this probability distribution may have an infinite peak at certain values, which would be the observed ones [99]. This would imply that wormholes would dress the bare constants of nature and therefore, when one is using a probe of certain (low-energy dressed) mass/size, it would behave not as a particle with these attributes but with the bare ones when probing small distances, so that our measurement would have an intrinsic uncertainty about small scale physics, this uncertainty being greater and greater as the distance becomes smaller and smaller.

\section{CONCLUSION}

Throughout this paper, we have displayed a series of arguments in quantum gravity that lead to the existence of a minimum length, a resolution limit in any experiment. This means that the notions of distance, of causality and any other notion based on a metric structure lose their meaning at the Planck's scale. This idea is very old and, in fact, can be traced back to the first third of this century. What is remarkable is that so many different analyses based on different ways of looking at the problem of quantum gravity give the same prediction about the nature of spacetime at the small-distance scale. The presence of a lower bound to the uncertainty of distance measurements seems to be a model-independent feature of quantum gravity. It is a direct consequence of the physical laws that affect the spacetime stucture. They can be summarized in the three following well-know statements: 
(i) the uncertainty principle, (ii) the speed of light is finite and constant, and (iii) the equivalence principle.

\section{ACKNOWLEDGMENTS}

I am very grateful to Jonathan Halliwell and Max Bañados for fruitful discussions and suggestions and for a critical reading of the manuscript. I also thank Alex Miković and Roya Mohayaee for useful conversations. The author was supported by a joint fellowship from the Ministerio de Educación y Ciencia (Spain) and the British Council. 


\section{REFERENCES}

[1] C. J. Isham, gr-qc/9310031, IC-TP/93-94/1 (1993). Prima facie questions in quantum gravity.

[2] V. B. Berestetskiǐ, E. M. Lifshitz and L. P. Pitaevskiǐ, Relativistic Quantum Theory (Pergamon, Oxford, 1971).

[3] W. Heisenberg, Z. Phys. 110, 251 (1938). The limits of the applicability of quantum theory up to now.

[4] T. D. Newton and E. P. Wigner, Rev. Mod. Phys. 21, 400 (1949). Localized states for elementary systems.

[5] J. A. Wheeler, in Relativity, Groups and Topology, eds. B. S. DeWitt and C. DeWitt (Gordon and Breach, London, 1964). Geometrodynamics and the issue of the final state.

[6] M. A. Markov, Preprint P-0187 of the Inst. Nucl. Res., Moscow (1980). On quantum violation of topology in small spatial regions.

[7] M. A. Markov, Preprint P-0208 of the Inst. Nucl. Res., Moscow (1981). On the maximon and the concept of elementary particle.

[8] H.-H. Borzeszkowski and H.-J. Treder, The Meaning of Quantum Gravity (Reidel, Dordrecht, 1988).

[9] T. Padmanabhan, T. R. Seshadri and T. P. Singh, Int. J. Mod. Phys. A1, 486 (1986). Uncertainty principle and the quantum fluctuations of the Schwarzschild light cones.

[10] V. Daftardar and N. Dadhich, Int. J. Mod. Phys. A1, 731 (1986). Uncertainty principle and the quantum fluctuation of the light cones in the static space-times.

[11] Chung-I Kuo and L. H. Ford, Phys. Rev D47, 4510 (1993). Semiclassical gravity theory and quantum fluctuations.

[12] C. A. Mead, Phys. Rev. 135, B849 (1964). Possible connection between gravitation and fundamental length.

[13] N. Itzhaki, Phys. Lett. B328, 274 (1994). Time measurement in quantum gravity.

[14] T. Padmanabhan, Ann. Phys. (NY) 165, 38 (1985). Physical significance of Planck's length.

[15] T. Padmanabhan, Gen. Rel. Grav. 17, 215 (1985). Planck length as the lower bound to all physical length scales.

[16] T. Padmanabhan, Class. Q. Grav. 3, 911 (1986). Role of general relativity in uncertainty principle.

[17] T. Padmanabhan, Class. Q. Grav. 4, L107 (1987). Limitations on the operational definition of space-time events and quantum gravity.

[18] J. Greensite, Phys. Lett. B255, 375 (1991). Is there a minimum length in $D=4$ lattice quantum gravity?

[19] J. V. Narlikar and T. Padmanabhan, Phys. Rep. 100, 151 (1983). Quantum cosmology via path integrals.

[20] B. A. Berg, B. Krishnan, M. Katoot, Nucl. Phys. B404, 359 (1993). Asymptotic freedom and Euclidean quantum gravity.

[21] B. A. Berg, B. Krishnan, Phys. Lett. B318, 59 (1993). Phase structure of SU(2) lattice gauge theory with quantum gravity.

[22] G. Feinberg, R. Friedberg, T. D. Lee, H. C. Ren, Nucl. Phys. B245, 343 (1984). Lattice gravity near the continuum limit. 
[23] M. Kato, Phys. Lett. B245, 43 (1990). Particle theories with minimum observable length and open string theory.

[24] K. Konishi, G. Paffuti and P. Provero, Phys. Lett. B234, 276 (1990). Minimum physical length and the generalized uncertainty principle in string theory.

[25] G. Veneziano, Europhys. Lett. 2, 199 (1986). A stringy nature needs just two constants.

[26] D. Amati, M. Ciafaloni and G. Veneziano, Phys. Lett. B216, 41 (1989). Can spacetime be probed below the string size?

[27] T. Yoneya, Mod. Phys. Lett. A4, 1587 (1989). On the interpretation of minimal length in string theories.

[28] R. Guida, K. Konishi and P. Provero, Mod. Phys. Lett. A6, 1487 (1991). On the short distance behaviour of string theory.

[29] P. F. Mende, hep-th/9210001, Brown-HET-875 (1992). String theory at short distance and the principle of equivalence.

[30] A. Strominger, hep-th/9110011, UCSB-TH-91-41 (1991). Quantum gravity and string theory: what we have learned.

[31] V. P. Nair, A. Shapere, A. Strominger and F. Wilczek, Nucl. Phys. B287, 402 (1987). Compactification of the twisted heterotic string.

[32] R. Brandenberger and L. Vafa, Nucl. Phys. B316, 391 (1989). Superstrings in the early universe.

[33] Y. I. Kogan, JETP Lett. 45, 709 (1987). Vortices on the world sheet and string's critical dynamics.

[34] Y. I. Kogan, Mod. Phys. Lett. A6, 501 (1991). The $R \rightarrow \alpha^{\prime} / R$ spacetime duality from the $2+1$ point of view.

[35] P. S. Aspinwall, B. R. Greene, D. R. Morrison, Nucl. Phys. B420, 184 (1994). Measuring small distances in $N=2$ sigma models.

[36] P. S. Aspinwall, hep-th/9404060, IASSNS-HEP-94-19 (1994). Minimum distances in nontrivial string target spaces.

[37] I. Klebanov and L. Susskind, Nucl. Phys. B309, 175 (1988). Continuum string theories from discrete field theories.

[38] D. Gross and P. F. Mende, Phys. Lett. B197, 129 (1987). The high energy behaviour of string scattering amplitudes.

[39] D. Gross and P. F. Mende, Nucl. Phys. B303, 407 (1988). String theory beyond the Planck scale.

[40] J. J. Atick and E. Witten, Nucl. Phys. B310, 291 (1988). The Hagedorn transition and the number of degrees of freedom of string theory.

[41] C. Rovelli and L. Smolin, Phys. Rev. Lett. 61, 1155 (1988). Knot theory and quantum gravity.

[42] C. Rovelli and L. Smolin, Nucl. Phys. B331, 80 (1990). Loop space representation of quantum gravity.

[43] C. Rovelli, Class. Q. Grav. 8, 1613 (1991). Ashtekar formulation of general relativity and loop space nonperturbative quantum gravity: a report.

[44] L. Smolin, in Quantum Gravity and Cosmology, eds. J. Pérez-Mercader et al. (World Scientific, Singapore, 1992). Recent developments in nonperturbative quantum gravity.

[45] C. Rovelli, Class. Q. Grav. 8, 317 (1991). Quantum reference systems.

[46] A. Ashtekar, C. Rovelli and L. Smolin, Phys. Rev. Lett. 69, 237 (1992). Weaving a 
classical metric with quantum threads.

[47] C. Rovelli, Nucl. Phys. B405, 797 (1993). A generally covariant quantum field theory and a prediction on quantum measurements of geometry.

[48] J. D. Bekenstein, Lett. Nuovo Cim. 11, 467 (1974). The quantum mass spectrum of the Kerr black hole.

[49] V. F. Mukhanov, JETP Lett. 44, 63 (1986). Are black holes quantized?

[50] Y. I. Kogan, JETP Lett. 44, 267 (1986). Quantization of the black hole mass in string theory.

[51] J. García-Bellido, hep-th/9302127, SU-ITP-93-4, IEM-FT-68/93 (1993). Quantum black holes.

[52] Y. Peleg, hep-th/9307057, BRX-TH-350 (1993). Quantum dust holes. A statistical mechanics derivation of black hole thermodynamics.

[53] M. Maggiore, Phys. Lett. B304, 65 (1993). A generalized uncertainty principle in quantum gravity.

[54] J. Preskill, P. Schwarz, A. Shapere, S. Trivedi and F. Wilczek, Mod. Phys. Lett. A6, 2353 (1991). Limitations on the statistical description of black holes.

[55] S. Deser, Rev. Mod. Phys. 29, 417 (1957). General relativity and the divergence problem in quantum field theory.

[56] T. D. Lee, Phys. Lett. B122, 217 (1983). Can time be a discrete variable?

[57] T. Padmanabhan, Phys. Rev. Lett. 60, 2229 (1988). Acceptable density perturbations from inflation due to quantum gravitational damping.

[58] C. A. Mead, Phys. Rev. 143, 990 (1966). Observable consequences of fundamental length hypotheses.

[59] P. F. González-Díaz, Lett. Nuovo Cim. 41, 481 (1984). Small-distance derivatives.

[60] P. K. Townsend, Phys. Rev. D15, 2795 (1977). Small-scale structure of spacetime as the origin of the gravitational constant.

[61] M. Maggiore, Phys. Rev. D49, 5182 (1993). Quantum groups, gravity and the generalized uncertainty principle.

[62] M. Maggiore, Phys. Lett. B319, 83 (1993). The algebraic structure of the generalized uncertainty principle.

[63] A. Kempf, hep-th/9311147, DAMTP-93-65 (1993). Uncertainty relation in quantum mechanics with group symmetry.

[64] A. Kempf, S. Majid, hep-th/9405067, DAMTP-94-33 (1994). Quantum field theory with non-zero minimal uncertainties in positions and momenta.

[65] N. Bohr and L. Rosenfeld, Det. Kgl. Danske Videnskabernes Selskab., Math.-fys. Medd. 12, 8 (1933); in Selected Papers of Lèon Rosenfeld, eds. Cohen and Stachel (Reidel, Dordrecht, 1979); in Quantum Theory and Measurement, eds. J. A. Wheeler and W. H. Zurek (Princeton University Press, Princeton, 1983). On the question of measurability of electromagnetic field quantities.

[66] L. Rosenfeld, Nucl. Phys. 40, 353 (1963). On quantization of fields.

[67] N. Bohr and L. Rosenfeld, Phys. Rev. D78, 794 (1950). Field and charge measurements in quantum electrodynamics.

[68] L. Rosenfeld, in Niels Bohr and the Development of Physics, ed. W. Pauli (Pergamon, London, 1955). On quantum electrodynamics.

[69] W. Heitler, The Quantum Theory of Radiation (Oxford University Press, New York, 3rd 
ed., 1954).

[70] A. Peres and N. Rosen, Phys. Rev. 118, 335 (1960). Quantum limitations on the measurements of the gravitational field.

[71] B. S. DeWitt, in Gravitation: An Introduction to Current Research, ed. L. Witten (Wiley, New York, 1962). The quantization of gravity.

[72] T. Regge, Nuovo Cim. 7, 215 (1958). Gravitational fields and quantum mechanics.

[73] H.-H. Borzeszkowski and H.-J. Treder, Found. Phys. 12, 1113 (1982). Quantum theory and Einstein's general relativity.

[74] H.-J. Treder, Found. Phys. 15, 161 (1985). The Planckions as the largest elementary particles and the smallest test bodies.

[75] L. Landau and R. Peierls, Z. Phys. 69, 56 (1931); in Collected Papers of Landau, ed. D. ter Haar (Gordon and Breach, New York, 1965); in Quantum Theory and Measurement, eds. J. A. Wheeler and W. H. Zurek (Princeton University Press, Princeton, 1983). Extension of the uncertainty principle to the relativistic quantum theory.

[76] M. B. Mensky, Phys. Lett. A155, 229 (1991). The action uncertainty principle in continuous quantum measurements.

[77] M. B. Mensky, Phys. Lett. A162, 219 (1992). The action uncertainty principle and quantum gravity.

[78] J. J. Halliwell, Phys. Rev. D48, 4785 (1993). Quantum mechanical histories and the uncertainty principle II. Fluctuations about classical predictability.

[79] L. Susskind, Phys. Rev. Lett. 71, 2368 (1993). String theory and the principle of black hole complementarity.

[80] L. Susskind, Phys. Rev. D49, 6606 (1994). Strings, black holes and Lorentz contraction.

[81] M. Maggiore, Phys. Rev. D49, 2918 (1994). Black hole complementarity and the physical origin of the stretched horizon.

[82] T. Jacobson, Phys. Rev. D44, 1731 (1991). Black-hole evaporation and ultrashort distances.

[83] L. Susskind, L. Thorlacius and J. Uglum, Phys. Rev. D48, 3743 (1993). The stretched horizon and black hole complementarity.

[84] L. Susskind and L. Thorlacius, Phys. Rev. D49, 966 (1994). Gedanken experiments involving black holes.

[85] G. 't Hooft, Nucl. Phys. B256, 727 (1985). On the quantum structure of a black hole.

[86] G. 't Hooft, Nucl. Phys. B335, 138 (1990). The black hole interpretation of strings.

[87] J. J. Halliwell, in Quantum Cosmology and Baby Universes, eds. S. Coleman et al. (World Scientific, Singapore, 1990). Introductory lectures on quantum cosmology.

[88] J. A. Wheeler, Ann. Phys. 2, 604 (1957). On the nature of quantum geometrodynamics.

[89] J. A. Wheeler, Geometrodynamics (Ac. Press, London, 1962).

[90] S. W. Hawking, Nucl. Phys. B144, 349 (1978). Spacetime foam.

[91] S. W. Hawking, Phys. Rev. D37, 904 (1988). Wormholes in spacetime.

[92] S. W. Hawking, Mod. Phys. Lett. A5, 145 (1990). Baby universes.

[93] S. W. Hawking, Mod. Phys. Lett. A5, 453 (1990). Baby universes II.

[94] A. Strominger, in Particles, Strings and Supernovae, eds. A. Jevicki and C. I. Tan (World Scientific, Singapore, 1989). Baby universes.

[95] S. W. Hawking and D.N. Page, Phys. Rev. D42, 2655 (1990). Spectrum of wormholes.

[96] L. J. Garay, Phys. Rev. D44, 1059 (1991). Quantum state of wormholes and path integral. 
[97] L. J. Garay, Phys. Rev. D48, 1710 (1993). Hilbert space of wormholes.

[98] S. W. Hawking, in General Relativity: An Einstein Centenary Survey, eds. S. W. Hawking and W. Israel, (Cambridge University Press, Cambridge, 1979). The path integral approach to quantum gravity.

[99] S. Coleman, Nucl. Phys. B310, 643 (1988). Why there is nothing rather than something: a theory of the cosmological constant.

[100] I. Klebanov, L. Susskind and T. Banks, Nucl. Phys. B317, 665 (1989). Wormholes and the cosmological constant.

[101] J. Preskill, Nucl. Phys. B323, 141 (1989). Wormholes in spacetime and the constants of nature. 